\title{
El rol de la función de transferencia de contraste en la formación de imágenes de resolución atómica de nanopartículas
}

\section{The role of the contrast transfer function in the formation of atomic resolution images of nanoparticles}

\author{
Carlos Angeles Chávez*
}

\begin{abstract}
High resolution transmission electron microscopy results can be quantitatively interpreted if an optimum adjustment is performed in the optical-electronic of the instrument during the atomic resolution images acquisition. This work qualitatively describes the principles of imaging formation by using the theory of electron diffraction and electronic-optics. At the end, the process is summarized through two parameters: contrast transfer function (CTF) and defocus of the image. The result is the modulation of the amplitude and phase in the CTF in order to obtain stronger phase contrast on the $d$-spacing of the nanoparticle under observation. Brighter or darker contrasts can be achieved from Scherzer focus and at values of bigger negative defocus. In this way, dark contrasts of $\mathrm{WO}_{\mathrm{x}}$ clusters on crystalline lattice of bright contrast corresponding to the $m-\mathrm{ZrO}_{2}$ nanoparticle through a controlled and precise manipulation of the defocus was evidenced.
\end{abstract}

KEYWORDS: high resolution transmission electron microscopy (HRTEM), atomic resolution, CFT, nanoparticle, phase contrast.

RESUMEN: Los resultados de microscopía electrónica de transmisión de alta resolución pueden ser cuantitativamente interpretados cuando se realizan los ajustes necesarios de la ópticaelectrónica del instrumento para la adquisición de las imágenes de resolución atómica. En este trabajo se describen cualitativamente los principios de la formación de imagen en términos de la difracción de electrones y de la óptica electrónica. Al final, el proceso se resume a dos parámetros: la función de transferencia de contraste (FTC) y el desenfoque de la imagen del objeto. El resultado es el control preciso de la modulación de la amplitud y la fase de la FTC a través del desenfoque para conseguir contraste de fase más fuerte e intensos en los espaciados interplanares de la red cristalina de la partícula bajo estudio. Contrastes intensos brillantes u oscuros se pueden lograr a partir del foco de Scherzer hacia valores más negativos. De esta manera, se logró evidenciar clústeres de $\mathrm{WO}_{\mathrm{x}}$ de contrastes oscuros sobre líneas de red de contraste brillante de la $\mathrm{m}-\mathrm{ZrO}_{2}$, modulando la fase y amplitud de la FTC a través de la manipulación controlada y precisa del foco.

PALABRAS CLAVE: microscopía electrónica de transmisión (MET), imágenes, resolución atómica, nanopartícula, contraste de fase.

Recibido: 28 de enero de 2019. Aceptado: 28 de abril de 2020 .

* Gerencia de Desarrollo de Materiales y Productos Químicos, Instituto Mexicano del Petróleo. Eje Central Lázaro Cárdenas 152, C. P. 07730, Ciudad de México, México.

Correo electrónico: cangeles@imp.mx | cach6906@gmail.com 


\section{Introducción}

La microscopía electrónica de transmisión de alta resolución (HRTEM) es una técnica que permite observar los detalles estructurales de la materia sólida a escala atómica proporcionando un conocimiento directo de la estructura de la materia en forma de una imagen (Urban, 2008; Thomas, 2017; Su, D. 2017; Ophus, C. 2019, Van Tendeloo et al., 2012).

Actualmente, los microscopios son capaces de resolver distancias tan pequeñas como $1 \AA$, lo suficiente como para separar las columnas atómicas en un cristal (Lentzen, 2008; Jia y Urban, 2004; Borodko et al., 2012). A diferencia de la microscopía de barrido de tunelamiento (STM), la cual es su principal competidor en resolución pero que solo resuelve la superficie del objeto, la HRTEM es un método de proyección cuya señal generada es sensitiva al volumen del objeto y la información es transferida a una imagen bidimensional (Barthel, 2008; Peng et al., 2008; Stroppa, et al., 2009; Kleebe, et al., 2010). Desafortunadamente, la interpretación no es tan sencilla como en la microscopía óptica de luz tradicional. La imagen bidimensional, capturada por la cámara (charge-coupled device) CCD, es producto de la interacción del haz de electrones-átomos del objeto y los efectos de incoherencia y aberraciones ópticas de la lente objetiva (Wen-Kuo et al., 2004; Sibarita, 2005; Bals et al., 2006). Así que, la información capturada está codificada y no es directamente interpretable o intuitiva.

Durante las últimas décadas, se ha invertido una gran cantidad de esfuerzo científico para resolver el problema de la interpretación. El resultado de mayor logro ha sido la simulación de imágenes (Peng, et al., 2008; Stroppa, et al., 2009; Wen-Kuo et al., 2004). Es una de las técnicas que toma en cuenta la mecánica cuántica del proceso de la interacción entre electrones y átomos, y la transferencia del contraste óptico-electrónico para la formación de la imagen. La mayoría de las técnicas únicamente proporcionan una solución parcial o nula a la primera causa del problema, es decir, la mecánica cuántica (Sibarita, 2005; Hsieh, et al., 2007).

En la técnica de simulación de imágenes (Horiuchi y He, 2000; Thust et al., 1996; Hosokawa et al., 2015), una imagen teórica es calculada a partir de una estructura atómica propuesta. Sobre la base de una evaluación subjetiva entre la imagen experimental y la imagen calculada, se determina la calidad del ajuste y el modelo de estructura propuesto puede ser aceptado o rechazado. Sin embargo, para estructuras de objetos muy complejos o aperiódicos (Thust et al., 1996) esta técnica se vuelve tediosa o incluso con la cual es imposible conseguir un resultado aceptable debido a la falta total del conocimiento inicial de la estructura desconocida. Aun con esta desventaja, la simulación de imágenes, por tener en cuenta el complejo proceso de dispersión dentro del objeto, sigue siendo una herramienta indispensable para un análisis cuantitativo con mayor profundidad en las imágenes de HRTEM. Esto conlleva a que, para lograr una interpretación intuitiva de la imagen, sea necesario tomar en 
cuenta tanto el formalismo de la difracción como el formalismo de la formación de imágenes (Takai et al., 1997; Danev et al., 2002; Lentzen et al., 2002; Brent y Howe, 2008). Las teorías de difracción de electrones e imagen electrónica describen cómo se genera la información de la estructura del objeto dentro del objeto y luego, al final, cómo se transfiere al detector de imagen. Ambas teorías son bastante complejas, así que solo sus conceptos básicos de forma descriptiva serán abordados en el marco de este trabajo.

En un microscopio, el efecto combinado del desenfoque, aberraciones ópticas y aperturas físicas, limita tanto la resolución del instrumento como el contraste en la imagen (Takai et al., 1997). Hoy en día, es relativamente fácil obtener imágenes con detalles a escala atómica, sin embargo, relacionar la imagen con su estructura atómica es a menudo difícil. A menos que la imagen obtenida sea bajo ciertas condiciones durante la manipulación del instrumento. Análisis de estas condiciones y su implicación en la imagen grabada, son el objetivo de este estudio.

\section{Principio de formación de imagen de resolución atómica}

El principio básico de formación de imagen en microscopía electrónica de transmisión (MET) sigue un esquema directo como lo representa el diagrama de la figura 1. Un haz de electrones con longitud de onda $\lambda$-que no está localizado en el espacio para que cada electrón pueda considerarse como un ente aislado- incide como una onda plana sobre un plano de columnas atómicas de la red cristalina. Por simplicidad, se considerará exclusivamente una fila de átomos pero en realidad es un arreglo tridimensional.

FIGURA 1. Diagrama esquemático de la formación de imagen.

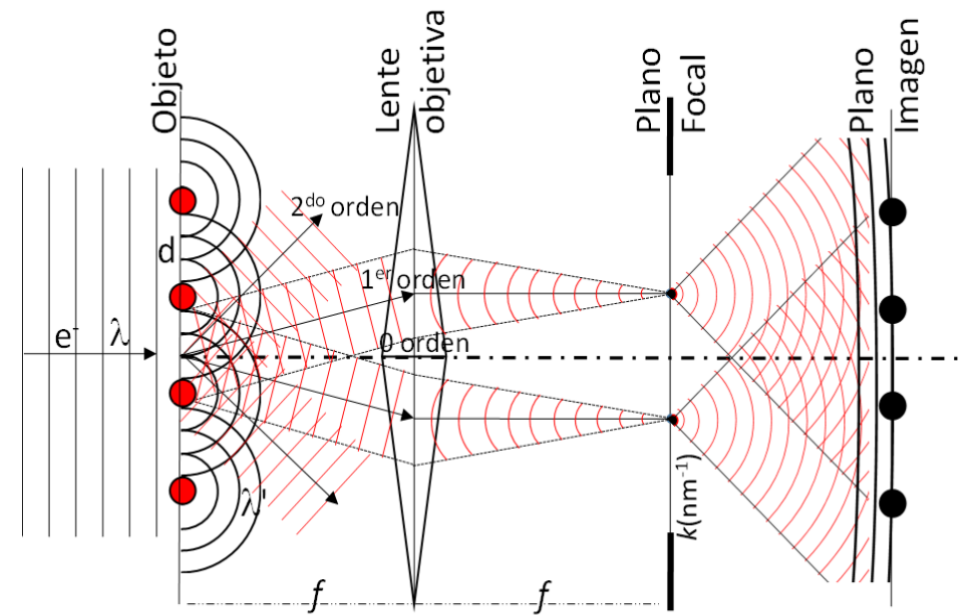

Fuente: Elaboración del autor. 
Durante la interacción, cada átomo de cada columna atómica produce ondas secundarias que interfieren con las otras ondas secundarias generadas por los átomos vecinos también. Estas interacciones múltiples finalmente producen una serie de interferencia de ondas constructivas y destructivas (Brent y Howe, 2008 ), y solo es posible si el cristal es lo suficientemente delgado, $10 \mathrm{~nm}$ de espesor como máximo, para permitir el paso de los electrones transmitidos y dispersados. Los máximos de las ondas secundarias entran en fase entre sí en una dirección bien definida. El caso más obvio es la dirección de la onda del electrón paralela a la onda entrante que corresponde al haz directo (no difractado) después de pasar el objeto. Este es identificado como un máximo orden cero. Otras líneas de máximos forman también planos de interferencia constructiva y ocurren en otras direcciones a un ángulo bien definido por el espaciado entre átomos del cristal y la longitud de onda de los electrones incidentes. Son identificados como haz de primer orden, segundo orden, y así sucesivamente, dando lugar a un patrón de difracción ocurrida en un plano bidimensional. El haz de primer orden tiene el ángulo de difracción más pequeño, por lo tanto, distancia entre átomos más grande. En el haz de segundo orden, el ángulo incrementa y la distancia entre átomos decrece, y así sucesivamente.

En este punto, los electrones difractados han sido afectados tanto en la fase como en la amplitud y pueden ser descritos mediante una función de onda de salida $\Psi(\mathrm{r}, \mathrm{z})$ (Allen et al., 2004; Op de Beeck, et al., 1996; Op de Beeck y Van Dyck, 1996 ). Donde $r$ representa el plano del cristal perpendicular al haz de electrones incidentes y $z$ el espesor del cristal perpendicular a $r$. Esta función de onda también puede ser representada por una serie de Fourier (Barthel, 2008). Los máximos de interferencia constructiva transportan información sobre la estructura atómica del objeto debido a la atracción del electrón incidente con los potenciales positivos atómicos de los núcleos de los átomos y los canales a lo largo de las columnas atómicas de la red cristalina. Posteriormente, los máximos de interferencia inciden en la lente objetiva, proyectándolos en el plano posterior focal como un patrón de puntos.

Matemáticamente, la lente objetiva convierte la función de onda de salida (serie de Fourier) en una transformada de Fourier (TF) y desplazamiento de fase (Peng et al., 2008). Por lo tanto, la función de onda de salida después de la lente objetiva está representada por $\mathrm{I}(\theta)=\delta(\theta)+\Phi \mathrm{K}(\theta)$ (Op de Beeck y Van Dyck, 1996), donde $\delta$ representa la onda de electrones trasmitidos, $\theta$ el ángulo de dispersión entre la onda de electrones dispersados y la onda de electrones transmitidos, $\Phi$ representa la TF de la fase de la función de onda y $\mathrm{K}(\theta)$ el desplazamiento de fase incurrido por las aberraciones de la lente objetiva. $K(\theta)$ es conocido también como función de transferencia de contraste (FTC), la cual modula la amplitud y la fase del patrón de difracción de electrones formado en el plano focal de la lente objetiva. 
Siguiendo el proceso de formación de imagen, el patrón de puntos formado en el plano focal es proyectado al plano imagen, que es el espacio real. Matemáticamente se describe como la inversa de la transformada de Fourier (ITF) de I $(\Theta)$ y se representa como el módulo cuadrado de la función de onda de electrones resultante (Op de Beeck y Van Dyck, 1996). Esta imagen, en el plano imagen, es finalmente ampliada por una serie de lentes electrónicas hasta la develación visual de las columnas atómicas, como la ilustrada en la figura 2a, en la cámara CCD de los microscopios modernos (De Ruijter et al., 1995). De este modo, la información sobre la estructura del objeto tridimensional queda transferido en una imagen bidimensional (Young-Min et al., 2006). De manera esquemática, la figura $2 \mathrm{~b}$ muestra la proyección de la información al plano imagen mostrando la intensidad de cada columna atómica. La variación en la intensidad es producto de las características de los átomos que la conforman tales como el ambiente químico y/o presencia de defectos.

Figura 2. a) Típica imagen de HRTEM proyectada bidimensionalmente, y, b) representación tridimensional de la distribución de intensidad de cada de punto brillante.
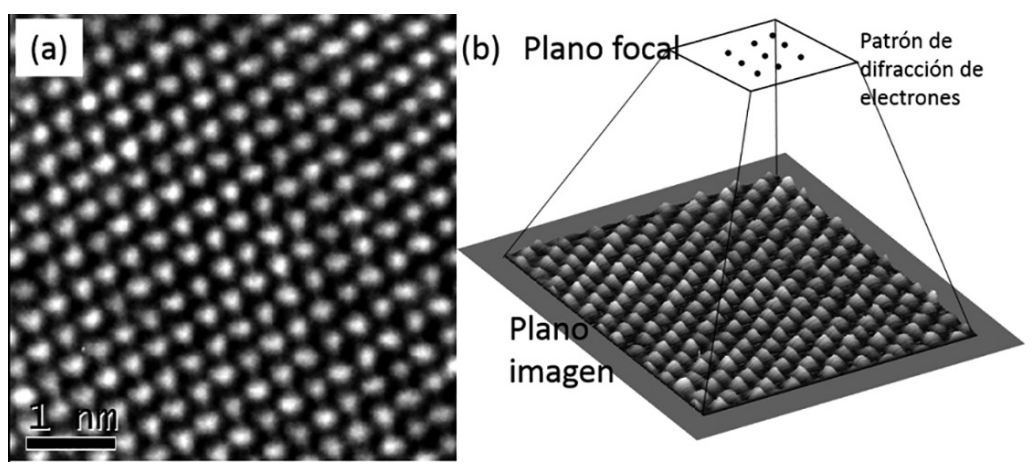

Fuente: Elaboración del autor.

Con la minimización de las aberraciones ópticas de las lentes condensadoras sobre el haz de electrones incidente, la lente objetiva se convierte en la parte fundamental del microscopio para la transferencia de la información contenida en el patrón de interferencia postrada en la salida del cristal en la imagen (Hawkes, 2015). Sin embargo, la intensidad de los puntos cambia con el grado de desenfoque como lo ilustra la imagen de HRTEM de la figura 3a y c. Misma área de observación del cristal tomadas a dos diferentes grados de desenfoque. El perfil de intensidad de una de las filas de puntos brillantes, en la figura $3 b$, decrece apreciablemente con el cambio de foco en la figura $3 \mathrm{~d}$, marcado por las flechas negras. Por consiguiente, es de extrema importancia fijar criterios en el grado de desenfoque para grabar la información más real del objeto bajo análisis en la imagen. 
Mundo Nano | ARTículos DE INVESTIGACión | www.mundonano.unam.mx

13(25), 9-27, julio-diciembre 2020 | https:// doi.org/10.22201/ceiich.24485691e.2020.25.69617

Carlos Angeles Chávez

Figura 3. a) y c) Imágenes de HRTEM de una partícula de $m-\mathrm{ZrO}_{2}$ tomadas a diferente desenfoque. b) y d) Perfil de intensidad de cada columna atómica en el mismo plano cristalino mostrando los cambios de intensidad con el desenfoque.
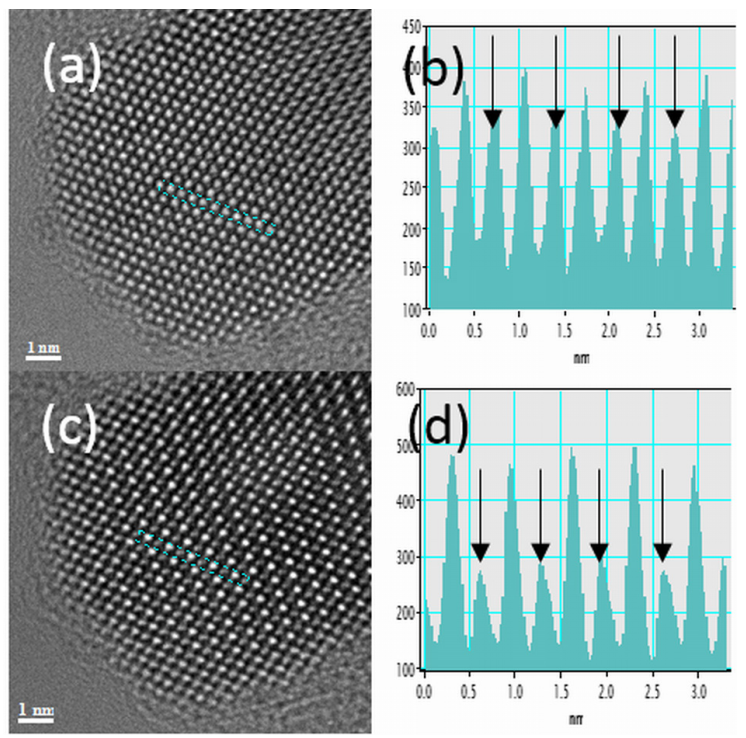

Fuente: Elaboración del autor.

\section{Función de transferencia de contraste}

Como se mencionó en el párrafo anterior, conseguir plasmar de forma más fiel en la imagen la información del cristal contenida en la función de onda del plano de salida después de cruzar la lente objetiva es de vital importancia. Así, la función de onda del plano de salida, fuertemente modificado durante su transferencia a través del sistema óptico del microscopio electrónico, es descrita por la FTC en el marco de un formalismo de onda-óptica por las denominadas aberraciones ópticas de la lente y las propiedades de imagen de la lente objetiva (Yang et al., 2009; Tromp y Schramm, 2013); es independiente del objeto y proporciona un mecanismo conveniente para entender el proceso de formación de la imagen y el contraste o intensidad. Para un mejor entendimiento, la FTC puede ser formulada en términos de frecuencias espaciales $(k)$ o espacio recíproco (Hyeong-Seop et al., 2013). Así, $\theta$ puede ser el producto $\lambda k y$, entonces, la FTC es descrita por $\mathrm{K}(k)=\mathrm{A}(k) \mathrm{E}(k) \operatorname{Sen}[\mathrm{X}(k)]$ donde $\mathrm{A}(k)$ es la función apertura que corta los haces dispersados arriba de un cierto ángulo crítico; $\mathrm{E}(k)$ la función envoltura que describe la atenuación de la onda para frecuencias espaciales altas; $\mathrm{X}(k)$ representa la función de las aberraciones del sistema óptico electrónico dado por $\pi C_{s} \lambda^{3} k^{4} / 2-\pi \Delta f \lambda k^{2}$ donde $C_{s}$ es el coeficiente de aberración esférica, $\lambda$ la longitud de onda del electrón y $\Delta f$ el desenfoque. En el MET, la medición y control del grado de desenfoque que puede lograrse es de una precisión tal que los cambios en la forma de la FTC (Presenza-Pitman, 
2009) y la modificación de la intensidad y nitidez de las frecuencias espaciales (k) son fácilmente detectables.

En un microscopio, el foco ideal produce una imagen de mínimo contraste, clara indicación de ausencia de fase del objeto. En el MET, dado que las aberraciones de la lente funcionan como una placa de fase (Nagayama y Danev, 2008) en el plano focal posterior del sistema óptico, difícilmente se obtiene una imagen de mínimo contraste con ausencia de fase en el foco ideal en una muestra cristalina. En la imagen, se registran tenuemente los máximos de la interferencia constructiva de las ondas generadas a la salida del objeto, como lo ilustra la figura 4a.

Figura 4. Imágenes de HRTEM de una partícula c-Ni- $\mathrm{ZrO}_{2}$ tomadas: a) desenfoque de mínimo contraste, y, b) desenfoque de máximo contraste.

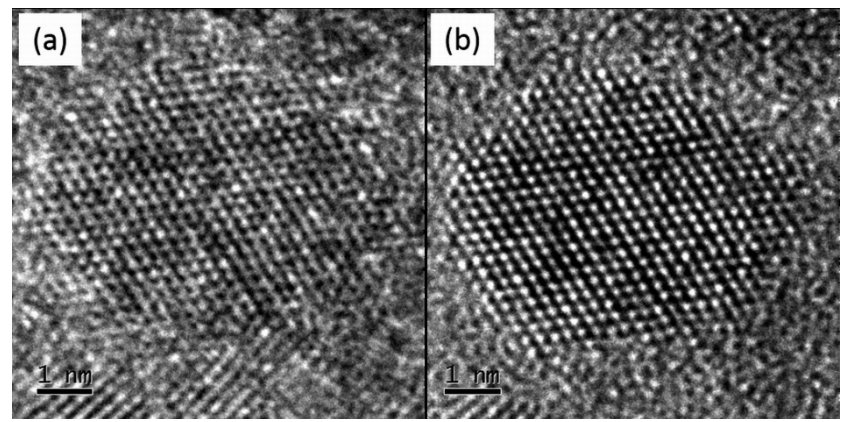

Nota: 4a: imagen adquirida en condiciones de foco, mínimo contraste, que contrasta fuertemente con la imagen ilustrada en la figura $4 \mathrm{~b}$, tomada a bajo foco en condiciones de máximo contraste brillante. Fuente: Elaboración del autor.

La generación de imágenes de máximo contraste ha sido ampliamente estudiada en microscopía de contraste de fase (Zhu et al., 1997) y la descripción del mecanismo para la ganancia de máximo contraste será utilizada para explicar los resultados desplegados en la figura 4. En el microscopio electrónico de transmisión, a condición de foco $\Delta f=0$, la lente objetiva (pieza polar) opera con una corriente eléctrica óptima (Barthel, 2008) y los puntos de difracción se localizan exactamente en el plano focal proyectando un frente de onda plano al plano imagen como se ilustra en la figura 1. Bajo estas condiciones, el contraste es mínimo o equivalente en cualquier parte de la imagen y nula o mínima información es visualizada en la imagen capturada. Cuando se incrementa la corriente eléctrica de la lente objetiva, más allá de la corriente óptima, el frente de onda en el plano imagen se convierte en convergente como esquemáticamente lo ilustra la figura 5 a. El punto de difracción se mueve en dirección hacia la lente objetiva, indicando mayor excitación de la lente y operando en condiciones de mayor inestabilidad. La imagen del objeto se encontrará en una posición de desenfoque positivo o 
sobrefoco $\Delta f>0$. Si se disminuye la corriente, obtendremos una lente más relajada y de mayor estabilidad. El punto de difracción se alejará de la lente objetiva produciendo un frente de onda divergente y una imagen del objeto con menor afectación eléctrica se encontrará en la posición de desenfoque negativo o bajofoco $\Delta f<0$. Este desenfoque retrasa la onda en fase con respecto a la onda en el eje óptico permitiendo obtener un incremento en el grado de contraste (figura $5 b$ ). Una mejor representación de esta descripción en términos de un desfasamiento de una longitud de onda en el dominio de la frecuencia espacial se ilustra en la figura $5 c$.

Figura 5. Esquema de desenfoque que ayuda a visualizar la diferencia de fase en el objeto: a) sobrefoco; b) bajofoco, y, c) función de transferencia de contraste sin aberraciones ópticas.

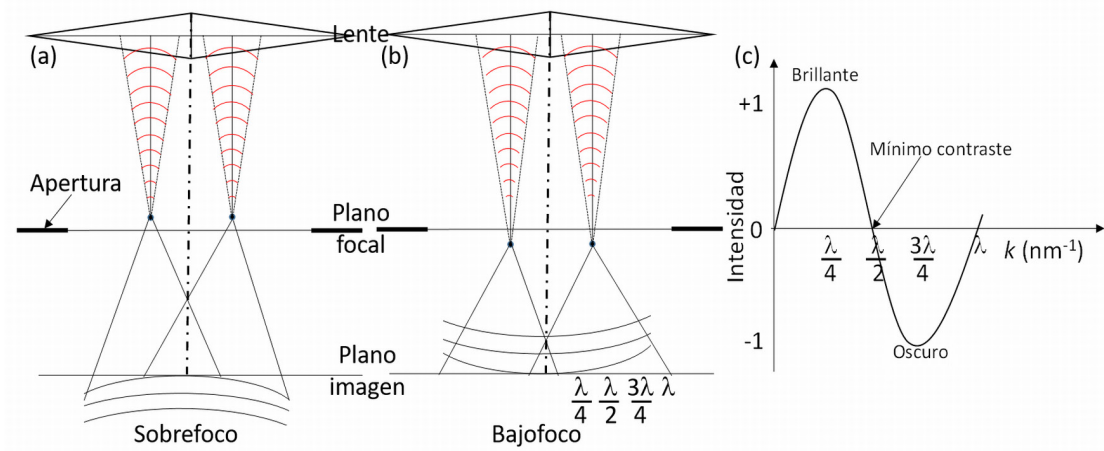

Fuente: Elaboración del autor.

Esta representación de la FTC que existe en el dominio de la frecuencia es-

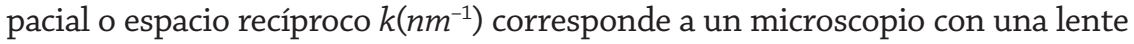
ideal, libre de aberraciones ópticas. En el origen, que corresponde al eje óptico del microscopio, la imagen del objeto se encuentra en foco y el valor de la FTC es cero, por lo tanto, el contraste en la imagen es mínimo. A medida que se va desenfocando la imagen, el frente de onda se va curvando y el plano imagen va detectando el desplazamiento de la fase hacia la cresta hasta alcanzar el máximo a un desenfoque de un cuarto de longitud de onda. El valor de la función de transferencia de contraste (FTC) es máximo y positivo generando una imagen de contraste brillante. Más incremento de desenfoque empezará a disminuir la brillantez hasta alcanzar el mínimo a la mitad de longitud de onda en el valor de la FTC igual a cero. Continuando con el desenfoque, la FTC empezará a tener valores negativos hasta alcanzar el mínimo a tres cuartos de longitud de onda y el contraste en la imagen será oscuro. Como lo ilustra el diagrama, el cambio gradual del desenfoque en uno u otro sentido cambiará sucesivamente el contraste de brillante a oscuro y viceversa. Sin embargo, en el microscopio electrónico de transmisión, la lente es demasiado imperfecta, tanto que las aberra- 
ciones ópticas modifican fuertemente la FTC (Op de Beeck y Van Dyck, 1996) de una lente ideal generando un perfil totalmente diferente al presentado en la figura 5c. La FTC real correspondiente a un microscopio electrónico de $200 \mathrm{kV}$ y Cs de $1 \mathrm{~mm}$ genera el perfil ilustrado en la figura 6.

Figura 6. FTC de un microscopio de $200 \mathrm{kV}$ y Cs de $1 \mathrm{~mm}$.

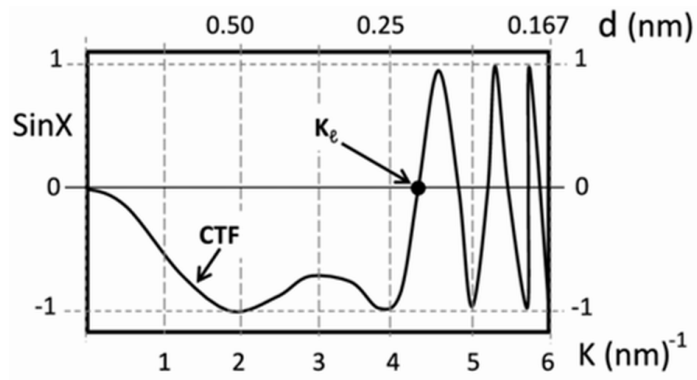

Fuente: Akhtar, 2012

En el gráfico se puede notar que la FTC inicia en cero. Inmediatamente después, se convierte en negativo, y representa el contraste de fase positivo que produce un fondo brillante con las características atómicas oscuras. Permanece negativo en un amplio rango de frecuencias espaciales $k$ proporcionando un amplio ancho de banda para resolver diferentes espaciados atómicos. En esta región, la información de la fase grabada en la imagen es directamente interpretable. Posteriormente, la FTC incrementa fuertemente su valor hasta intersectar con el eje X (Horiuchi y He, L. 2000). Aquí, en este valor de frecuencia espacial, la FTC fija el límite de resolución del instrumento y también fija el valor del foco en la imagen del objeto con el mínimo contraste ( Lentzen, 2008; Vulovic et al. 2012) como el ilustrado en la figura 4a. A partir de este punto, la FTC es oscilatoria y la información de la fase no es directamente interpretable. Las imágenes obtenidas bajo estas condiciones corresponden a condiciones de sobrefoco y la interpretación es realizada a través de simulación computarizada como lo podría ser el resultado mostrado en la figura $4 \mathrm{~b}$.

\section{Imágenes de HRTEM a partir de cálculos teóricos}

Puesto que la FTC modula la amplitud y la fase del patrón de difracción de electrones formado en el plano focal de la lente objetiva a través del desenfoque, es importante comprender su comportamiento con el espesor del cristal en una red cristalina. Esto se llevó a cabo a través de cálculos teóricos del modelo atómico de la estructura cristalina del Au con diferentes espesores. La figura 7 muestra la representación esquemática del modelo atómico utilizado orientado a lo largo de la dirección cristalográfica [110]. 
Figura 7. Modelo atómico de la estructura del oro vista en la dirección [110] perpendicular al haz de electrones incidente y su proyección tridimensional.

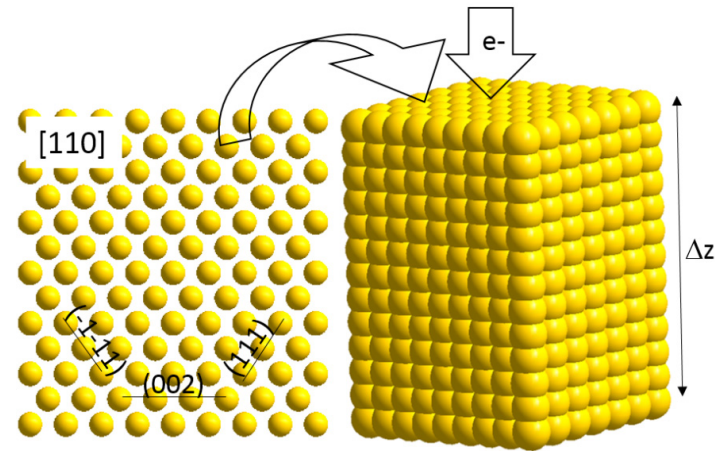

Fuente: Elaboración del autor.

Datos correspondientes a un microscopio electrónico de transmisión de $200 \mathrm{kV}$, con pieza polar de ultra alta resolución de $0.19 \mathrm{~nm}\left(k=5.26 \mathrm{~nm}^{-1}\right)$ punto a punto y $C$ s de $0.5 \mathrm{~mm}$ fueron alimentados al software Simulatem (Rodríguez y Beltrán, 2001) para calcular las imágenes de HRTEM.

Con este poder de resolución, los únicos espaciados interplanares que son resueltos de la red cristalina del oro corresponden a los planos (111) y (002), respectivamente. La revelación de estos planos cuando el cristal se orienta a lo largo de las direcciones $\langle 110\rangle$ y $\langle 001\rangle$, resuelven en la imagen columnas atómicas punto a punto. En otras orientaciones cristalográficas, el instrumento solo resuelve líneas de red. En el estudio, el cristal fue orientado a lo largo de la dirección [110] para visualizar las columnas atómicas de los planos (111) y (002) que deben ser proyectados en las imágenes simuladas. El patrón de puntos observado en la imagen debe reproducir el patrón de las columnas atómicas del modelo atómico en la dirección orientada para validar el cálculo teórico realizado por el software. Estos cálculos se realizaron para diferentes focos a un espesor de cristal definido observando la modulación de la FTC para cada cambio de foco. Al final se obtuvo una serie de imágenes con diferentes desenfoques, conocida como serie focal (Thust et al.,1996). Posteriormente, se seleccionaron las imágenes más nítidas de mayor contraste de puntos brillantes en fondo oscuro y puntos oscuros en fondo blanco basado en la teoría de la FTC del apartado anterior. La figura 8 despliega la serie de imágenes obtenidas a diferentes desenfoques y espesores reproduciendo con mayor nitidez las columnas atómicas del modelo atómico presentado en la figura 7.

De acuerdo con el análisis de desenfoque desarrollado por Scherzer (Lentzen, 2008; Horiuchi y He, 2000), el contraste de fase más alto es logrado en el llamado desenfoque de Scherzer que es $\Delta f_{S c}=-1.2(C s \lambda)=-31.6$ $\mathrm{nm}$; no obstante, la imagen con mayor contraste, a partir de un punto de 
Figura 8. Imágenes de HRTEM simuladas de la estructura de oro en la dirección [110] para diferentes desenfoques y espesores del cristal.

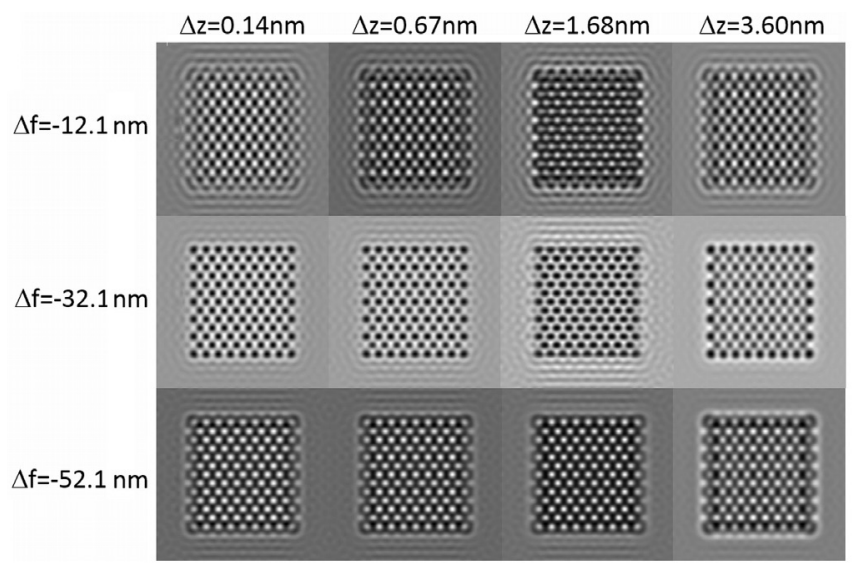

Fuente: Elaboración del autor.

vista cualitativo, es fijada por el espesor del cristal que difiere ligeramente del foco de Scherzer. Por lo tanto, en la figura se expone la imagen de mayor contraste obtenida a tres diferentes desenfoques para cada uno de los espesores evaluados: sobrefoco $(\Delta f=-12.1 \mathrm{~nm})$, cercano al foco de Scherzer $(\Delta f=$ $-32.1 \mathrm{~nm})$ y bajofoco $(\Delta f=-52.1 \mathrm{~nm})$.

La imagen correspondiente al cristal de espesor de $0.14 \mathrm{~nm}$, tomado a sobrefoco, no muestra un contraste de fondo definido. El patrón de puntos brillantes es similar al patrón de puntos oscuros haciendo difícil determinar el tipo de contraste correspondiente a la columna atómica. Una imagen similar fue obtenida para el espesor de $3.6 \mathrm{~nm}$ mientras que para los espesores $0.67 \mathrm{y}$ $1.68 \mathrm{~nm}$, los puntos brillantes parecen estar sobre un fondo oscuro y podrían ligeramente asociarse con las columnas atómicas. A condiciones de sobrefoco, el espesor tiene un efecto en el contraste mientras que a condiciones de bajofoco el efecto es imperceptible como se describirá a continuación. A desenfoque $\Delta f=-32.1 \mathrm{~nm}$, claramente se puede visualizar que las columnas atómicas corresponden a los puntos negros sobre fondo blanco, mientras que en las imágenes a bajofoco de $\Delta f=-52.1 \mathrm{~nm}$, las columnas atómicas son brillantes sobre un fondo obscuro. Así que a desenfoques de $\Delta f=-32.1$ y $-52.1 \mathrm{~nm}$, cada punto generado en la imagen representa una columna atómica del modelo para los diferentes espesores del cristal. Como se aprecia en la imagen, el grado de nitidez de la imagen cambia ligeramente con el espesor, por lo cual la presentación de estos resultados sugiere encontrar el mejor grado de desenfoque que proyecte la imagen con mayor grado de nitidez y contraste. Por ejemplo, los mejores contrastes para un espesor de $3.6 \mathrm{~nm}$ (imágenes no mostradas) se encontraron a $\Delta f=-35.1 \mathrm{~nm}$ para puntos oscuros, $\mathrm{y}-55.1 \mathrm{~nm}$, para puntos claros.

Los contrastes observados en las diferentes imágenes de la figura 8 pueden ser mejor comprendidos analizando el perfil de la FTC y los espaciados inter- 
planares en las gráficas de FTC de la figura 9. En particular para el espesor del cristal de $0.14 \mathrm{~nm}$. Los planos atómicos (111), (002) y (220) de la estructura del Au fueron insertados sobre el eje de la frecuencia espacial $k$ para visualizar el valor de la FTC al desenfoque evaluado y su interacción con el espaciado recíproco de dichos planos. Las gráficas claramente van ilustrando la modulación del contraste y la amplitud de la FTC en función del desenfoque. En el sobre foco, $\Delta f=-12.1 \mathrm{~nm}$, la FTC es positiva cuando intersecta con el espaciado reciproco del plano (111) y negativo con la intersección del plano (002). Esto podría explicar el patrón de contrastes claros-oscuros observado en la imagen. A $\Delta f=-32.1 \mathrm{~nm}$, la FTC es negativa en la intersección con ambos planos, por eso en la imagen se observan columnas atómicas oscuras sobre un fondo blanco. A $\Delta f=-52.1 \mathrm{~nm}$, la FTC es positiva en la intersección con ambos planos, proyectando en la imagen puntos brillantes sobre un fondo oscuro.

Figura 9. Gráficas de FTC para cada uno de los desenfoques utilizados para la simulación de las imágenes de HRTEM.

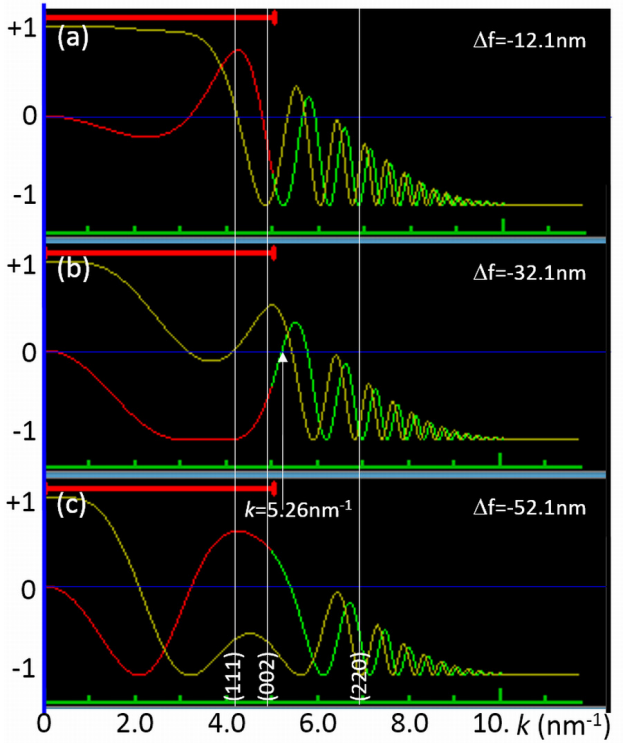

Fuente: Elaboración del autor.

\section{Imágenes experimentales de HRTEM de contrastes claros y oscuros}

La FTC real modulando la amplitud y la fase del patrón de difracción de electrones en el microscopio electrónico de transmisión JEM2200FS de 200kV de voltaje de aceleración y pieza polar de alta resolución, que resuelve punto a punto $0.19 \mathrm{~nm}$, es revelado a través de la trasformada de Fourier de las 
imágenes de HRTEM del soporte polimérico amorfo tomadas a diferentes desenfoques. Durante la adquisición de las imágenes, el soporte se mantuvo lo suficientemente estable para lograr medir con suficiente precisión el grado de desenfoque. Los resultados obtenidos se ilustran en la figura 10. Cuatro imágenes a desenfoques de $\Delta f=105,0,-64$ y $-160 \mathrm{~nm}$ muestran en sus TF el comportamiento de la FTC dentro del rango de frecuencias espaciales del límite de resolución del microscopio.

Figura 10. a-d) Imágenes de HRTEM tomadas del soporte polimérico de una rejilla de cobre de TEM a diferentes desenfoques. e-h) Transformadas de Fourier de las imágenes de HRTEM mostrando el comportamiento de la FTC con el desenfoque.

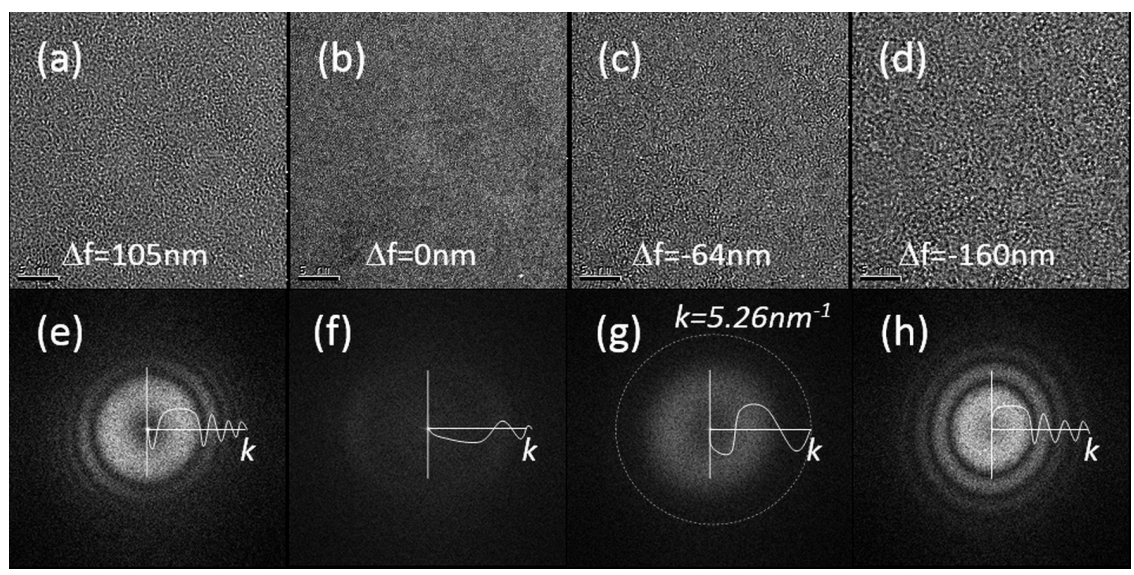

Fuente: Elaboración del autor.

Cada una de las FTC muestra claramente el ancho de banda donde las frecuencias espaciales serán brillantes (FTC positiva) y donde serán oscuras (FTC negativa) para cada grado de desenfoque de este espécimen en particular.

De acuerdo con los resultados de las imágenes teóricamente calculadas, insta tomar imágenes siempre a una menor excitación de la lente, es decir, a bajofoco $\Delta f<0$. La menor inestabilidad inducida por la corriente a la lente conducirá a la formación de una imagen más real de la estructura cristalina con mayor resolución y mejor nitidez (Barthel, 2008). Por lo tanto, es recomendable obtener imágenes a bajofoco por debajo del foco del límite de resolución como los ilustrados en la figura $10 \mathrm{~g}$ para puntos oscuros y figura $10 \mathrm{~h}$ para puntos brillantes. Dependiendo del espesor del espécimen o nanopartícula y sus espaciados interplanares, se permitirá determinar el grado de bajofoco óptimo para conseguir que las columnas atómicas estén dentro de un rango de ancho de banda amplio, donde la FTC sea positiva o negativa, para que los contrastes de las columnas atómicas sean oscuros o brillantes y conseguir una interpretación más directa de la imagen. Para algunos casos —el 
espaciado interplanar de la red cristalina del espécimen-, la FTC puede cubrir los espaciados interplanares tanto del lado positivo como del negativo permitiendo con el desenfoque a bajofoco obtener columnas atómicas oscuras para luego dar paso a las columnas brillantes a un mayor desenfoque como lo muestra la imagen de HRTEM de la figura 11. Defectos superficiales en la nanopartícula de zirconia claramente pueden caracterizarse en ambas imágenes.

Figura 11. Imágenes de HRTEM de una partícula de $\mathrm{c}-\mathrm{Ni}-\mathrm{ZrO}_{2}$ tomada a bajofoco de máximo contraste cuando la FTC es: a) negativo, columnas atómicas oscuras, y, b) positivo, columnas atómicas brillantes.

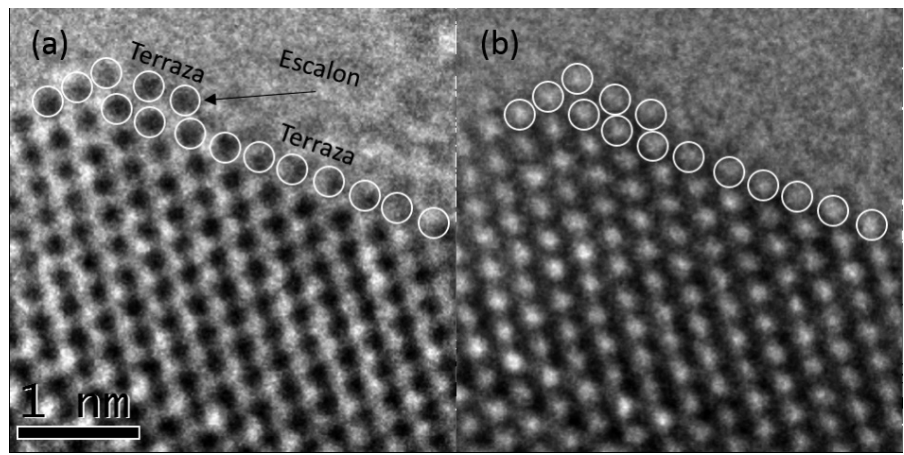

Nota: Defectos superficiales son claramente evidenciables. Fuente: Elaboración del autor.

\section{Imágenes experimentales de HRTEM en nanopartículas}

Basado en el conocimiento anteriormente descrito, imágenes experimentales de HRTEM evidenciando clústeres de $\mathrm{WO}_{\mathrm{x}}$ sobre partículas de zirconia fueron obtenidas mediante análisis MET a través de la modulación de la amplitud y de la fase de la FTC producida por la manipulación precisa del foco. Inicialmente, los átomos de tungsteno se encuentran dentro de la estructura como solución sólida estabilizando la fase tetragonal de la zirconia. Con la calcinación a $800{ }^{\circ} \mathrm{C}$ de esta muestra, el tungsteno es segregado y la zirconia tetragonal se transforma en monoclínica (Angeles-Chávez et al., 2006). El objetivo fue evidenciar directamente de la imagen, la presencia del tungsteno.

Los resultados de HRTEM obtenidos del análisis de la muestra calcinada a $800{ }^{\circ} \mathrm{C}$ son desplegados en la figura $12 \mathrm{a}-\mathrm{c}$. Las figuras $12 \mathrm{a}$ y $12 \mathrm{~b}$ corresponden a una partícula de zirconia tomada a dos diferentes grados de desenfoque. La primera imagen fue tomada después de haber modulado la amplitud y la fase de la FTC a través del foco en sentido negativo, hasta lograr que el ancho de banda fuera positivo en la frecuencia espacial de la red cristalina de la zirconia, generando las líneas de red brillantes. La segunda 
imagen, figura $12 b$, fue tomada después de la modulación de la FTC hacia valores de foco aún más negativos hasta que incluyera en la región negativa las líneas de red correspondientes a $\mathrm{WO}_{\mathrm{x}}$ y en la región positiva las líneas de red de la zirconia. La imagen proyectada reveló pequeñas regiones oscuras, marcadas con flechas blancas, sobre las líneas de red de la zirconia con baja resolución a este grado de desenfoque. Imagen de otra partícula, figura 12c, tomada bajo las mismas condiciones de modulación de la imagen de la figura $12 \mathrm{~b}$, reveló líneas de red de la zirconia de mayor espaciado interplanar mostrando nítidamente ambos contrastes: oscuros para los clústeres de $\mathrm{WO}_{\mathrm{x}} \mathrm{y}$ brillantes para la partícula de zirconia. La gran diferencia en resolución entre ambas partículas sugiere tomar en cuenta otros parámetros relacionados con sus características como la orientación cristalográfica, espesor, cristalinidad de las fases, dispersión de la fase, etc., para lograr proyectar la información contenida en el objeto en una imagen más interpretable como la ilustrada en la figura 12c.

Una validación de la interpretación de estos resultados experimentales se realizó a través del cálculo de la imagen proyectada de un modelo propuesto, es decir, clústeres de átomos de $\mathrm{W}$ y $\mathrm{O}$ de arreglo cristalino ortorrómbico de $\mathrm{WO}_{3}$ sobre un pequeño cristal de $m-\mathrm{ZrO}_{2}$. Los resultados obtenidos son desplegados en las figuras 12e-f, mostrando imágenes calculadas

Figura 12. a-c) Imágenes de HRTEM de partículas de $m-\mathrm{ZrO}_{2}$ mostrando la modulación del contraste de fase e intensidad a través del foco para evidenciar los clústeres de $\mathrm{WO}_{\mathrm{x}}$ de contraste oscuro sobre la red de $\mathrm{ZrO}_{2}$ de contraste brillante. d-e) Imágenes calculadas a diferentes grados de desenfoques a partir de un modelo atómico propuesto: Clústeres de $\mathrm{WO}_{\mathrm{x}}$ ortorrómbico sobre cristal de $\mathrm{m}-\mathrm{ZrO}_{2}$.

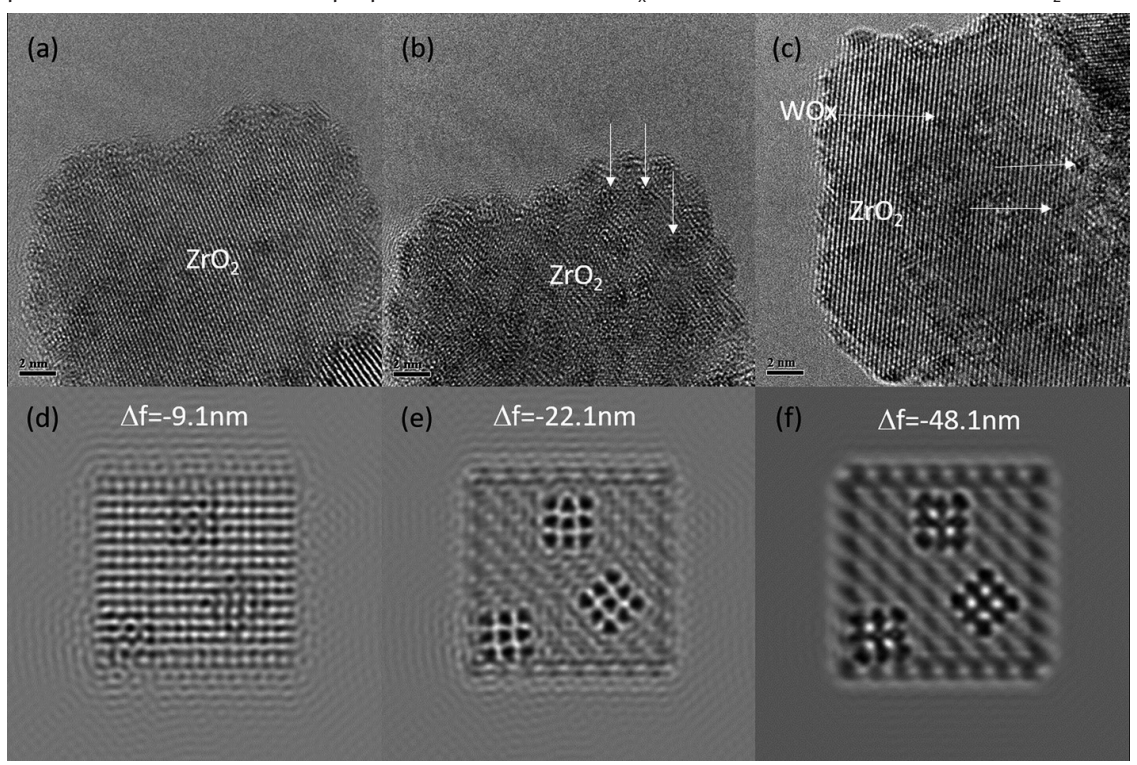

Fuente: Elaboración del autor. 
a $\Delta f=-9.1 \mathrm{~nm}, \Delta f=-22.1 \mathrm{~nm}, \mathrm{y}, \Delta f=-48.1 \mathrm{~nm}$. Las imágenes revelan cómo a través del desenfoque de la modulación de la amplitud y la fase de la FTC es posible develar los clústeres de WOx sobre el cristal de $m-\mathrm{ZrO}_{2}$ mediante la ganancia de contraste oscuro generado por el incremento del grado de desenfoque en el sentido negativo. Puntos oscuros correspondientes a los átomos de $\mathrm{W}$ de los clústeres de WOx son observados en las imágenes calculadas a $\Delta f=-22.1 \mathrm{~nm}$ y $\Delta f=-48.1 \mathrm{~nm}$ a diferencia de la imagen calculada a $\Delta f=-9.1 \mathrm{~nm}$, donde únicamente las líneas de red correspondiente a la $m-\mathrm{ZrO}_{2}$ fueron reveladas nítidamente y perdida en los desenfoques posteriores. Como puede verse, son resultados muy congruentes con los resultados de las imágenes experimentales de la figuras $12 \mathrm{a}$ y $12 \mathrm{~b}$. Por lo tanto, la modulación de la transferencia de amplitud y fase del objeto con cambios precisos de foco permite seleccionar las frecuencias que serán brillantes y las frecuencias que serán oscuras para proyectar la información más real contenida en el objeto bajo estudio. Sin embargo, el alto grado de desenfoque conduce irremediablemente a una disminución de la resolución del instrumento. Como resultado, aunque la morfología general de la muestra a escala nanométrica se puede deducir mediante imágenes bajo desenfoque, la información de resolución espacial más alta generalmente se pierde por lo que la imagen se deberá tomar en función de la información que se desea evidenciar.

\section{Conclusiones}

La FTC y el desenfoque de la imagen del objeto tienen un papel importante en la modulación de la amplitud y la fase del patrón de difracción de electrones formado en el plano focal de la lente objetiva modificando fuertemente el contraste de las frecuencias espaciales de la red cristalina. La generación de imágenes más interpretables implica modular la fase y amplitud de la FTC mediante un control preciso del grado de desenfoque en el sentido negativo del foco hasta lograr intersectar las frecuencias espaciales en el contraste deseado a partir del valor del foco de Scherzer. El desenfoque hacia el lado negativo del foco implica suministro de menor corriente eléctrica a la lente objetiva lo que conduce a una menor inestabilidad y una mejora en la imagen proyectada. La validez total de la interpretación de una imagen siempre será respaldada a través de la imagen calculada teóricamente a partir de un modelo atómico del cristal.

\section{Referencias}

Akhtar, S. (2012). Transmission electron microscopy of graphene hydrated biomaterials nanostructures: Novel techniques and analysis. Thesis dissertation, Faculty of Science and Technology, Uppsala University, Uppsala, Sweden. http://urn. kb.se/resolve?urn=urn:nbn:se:uu:diva-171991 
Allen, L. J., McBride, W., O’Leary, N. L., Oxley, M. P. (2004). Exit wave reconstruction at atomic resolution. Ultramicroscopy, (100)91. https://doi.org/10.1016/j. ultramic.2004.01.012

Angeles-Chávez, C., Cortés-Jácome, M.A., Torres-García, E., Toledo-Antonio, J. A. (2006). Structural evolution of $\mathrm{WO}_{3}$ nanoclusters on $\mathrm{ZrO}_{2}$. Journal Materials Research, 807. https://doi.org/10.1557/jmr.2006.0100

Bals, R. S., Van Aert, S., Van Tendeloo, G., Avila-Brande, D. (2006). Statistical estimation of atomic positions from exit wave reconstruction with a precision in the picometer range. Phys. Rev. Lett. (96): 096106. https://doi.org/10.1103/PhysRevLett.96.096106

Barthel, J. (2008). Ultra-precise measurement of optical aberrations for sub-angström transmission electron microscopy. Thesis dissertation, Von der Fakultät für Mathematik, Informatik und Naturwissenschaften der Rheinisch-Westfälischen Technischen Hochschule, Germany, N. p., Web. https://www.osti.gov/ etdeweb/biblio/21084262

Borodko, Y., Ercius, P., Pushkarev, V., Thompson, C., Somorjai, G. (2012). From single Pt atoms to Pt nanocrystals: Photoreduction of Pt2+ inside of a PAMAM dendrimer. J. Phys. Chem.Lett., (3): 236. https://doi.org/10.1021/jz201599u

Brent, F., Howe, J. M. (2008). Transmission electron microscopy and diffractometry of materials, 3a ed. Springer-Verlag, Berlín, Heidelberg. https://doi.org/10.1007/9783-540-73886-2

Danev, R., Okawara, H., Usuda, N., Kametani, K., Nagayama, K. (2002). A novel phase-contrast transmission electron microscopy producing high-contrast topographic images of weak objects. J. Biol. Phys., (28): 627. https://doi. org/10.1023/A:1021234621466

De Ruijter, W. J., Sharma, R., McCartney, M. R., Smith, D. J. (1995). Measurement of lattice-fringe vectors from digital HREM images: experimental precision. Ultramicroscopy, (57): 409. https://doi.org/10.1016/0304-3991(94)00166-K

Hawkes, P. W. (2015). The correction of electron lens aberrations. Ultramicroscopy, (156) A1. doi.org/10.1016/j.ultramic.2015.03.007

Horiuchi, S., He, L. (2000). High-resolution transmission electron microscopy, characterization of high Tc materials and devices by electron microscopy, Nigel D. Browning, Stephen J. Pennycook (eds.). Cambridge. https://doi.org/10.1017/CBO9780511534829

Hosokawa, F., Shinkawa, T., Arai, Y., Sannomiya, T. (2015). Benchmark test of accelerated multi-slice simulation by GPGPU. Ultramicroscopy, (15): 856. https:// doi.org/10.1016/j.ultramic.2015.06.018

Hsieh, W. K., Anderson, E. H., Benner, G., Park, M. J., Gómez, E.D., Balsara, N.P., Kisielowski, C. (2007). Contrast transfer function design by an electrostatic phase plate. Microsc. Microanal., (13): 2. https://doi.org/10.1017/S1431927607073874

Hyeong-Seop, J., Hyo-Nam, P., Jin-Gyu, K., Jae-Kyung, H. (2013). Critical importance of the correction of contrast transfer function for transmission electron microscopy-mediated structural biology. J. Anal. Sci. Technol., (4): 14. https:// doi.org/10.1186/2093-3371-4-14

Jia, C. L., Urban, K. (2004). Atomic-resolution measurement of oxygen concentration 
in oxide materials. Science (303): 2001. https://doi.org/10.1126/science.1093617

Kleebe, H-J., Lauterbach, S., Müller, M. (2010). Transmission electron microscopy (TEM). Bunsen-Magazin, (5): 168. https://www.yumpu.com/en/document/ read/17191295/bunsen-magazin-deutsche-bunsengesellschaft-fur-physikalische-

Lentzen, M. (2008). Contrast transfer and resolution limits for sub-angstrom highresolution transmission electron microscopy. Microsc. Microanal., (14): 16. https://doi.org/10.1017/S1431927608080045

Lentzen, M., Jahnen, B., Jia, C.L., Thust, A., Tillmann, K., Urban, K. (2002). High resolution imaging with aberration-corrected transmission electron microscopy, Ultramicroscopy, (92): 233. https://doi.org/10.1016/S0304-3991(02)00139-0

Nagayama, K., Danev, R. (2008). Phase contrast electron microscopy: Development of thin-film phase plates and biological applications. Phil. Trans. R. Soc. B, (363): 2153. https://doi.org/10.1098/rstb.2008.2268

Op de Beeck, M., Van Dyck, D. (1996). Direct structure reconstruction in HRTEM. Ultramicroscopy, (64): 153. https://doi.org/10.1016/0304-3991(96)00006-X

Op de Beeck, M., Van Dyck, D., Coene, W. (1996). Wave function reconstruction in HRTEM: the parabola method. Ultramicroscopy, (64): 167. https://doi. org/10.1016/0304-3991(96)00058-7

Ophus, C. (2019). Four-dimensional scanning transmission electron microscopy (4D-STEM): From scanning nanodiffraction to ptychography and beyond. Microsc. Microanal., (25): 563. https://doi.org/10.1017/S1431927619000497

Peng, Y., Oxley, M. P., Lupini, A. R., Chisholm, M. F., Pennycook, S. J. (2008). Spatial resolution and information transfer in scanning transmission electron microscopy,Microsc.Microanal.,(14):36.https://doi.org/10.1017/S1431927608080161

Presenza-Pitman, G. (2009). Determination of the contrast and modulation transfer functions for high resolution imaging of individual atoms. Thesis dissertation, Department of Physics, University of Toronto, Toronto, Ontario. https://www. researchgate.net/publication/253639776

Rodríguez, A. G., Beltrán, L. M. (2001). SimulaTEM: a program for the multislice simulation of image and diffraction patterns of non-crystalline objects. Rev. Latin Am. Metal. Mat. (21): 46. http://ve.scielo.org/scielo.php?script=sci_arttext\&pid $=$ S0255-69522001000200009

Sibarita, J-B. (2005). Deconvolution microscopy. Adv. Biochem. Engin./Biotechnol., (95) 201. https://doi.org/10.1007/b102215

Stroppa, D. G., Montoro, L. A., Beltrán, A., Conti, T. G., Da Silva, R. O., Andrés J., Longo, E., Leite, E. R., Ramírez, A. J. (2009). Unveiling the chemical and morphological features of $\mathrm{Sb}_{-} \mathrm{SnO}_{2}$ nanocrystals by the combined use of high-resolution transmission electron microscopy and ab initio surface energy calculations. J. Am. Chem. Soc., (131): 14544. https://doi.org/10.1021/ja905896u

$\mathrm{Su}, \mathrm{D}$. (2017). Advanced electron microscopy characterization of nanomaterials for catalysis. Green Energy \& Environment, (2): 70. https://doi.org/10.1016/j.gee.2017.02.001

Takai, Y., Ando, T., Ikuta, T., Shimizu, R. (1997). Principles and applications of defocus-image modulation processing electron microscopy. Scanning Microscopy, (11): 391. https://www.ecmjournal.org/smi/pdf/smi97-30.pdf 
Thomas, J. M. (2017). Reflections on the value of electron microscopy in the study of heterogeneous catalysts. Proc. R. Soc.A, (473): 20160714.https://doi.org/10.1098/ rspa.2016.0714

Thust, A., Coene, W. M. J., Op de Beeck, M., Van Dyck, D. (1996). Focal-series reconstruction in HRTEM: simulation studies on non-periodic objects. Ultramicroscopy, (64): 211. https://doi.org/10.1016/0304-3991(96)00011-3

Tromp, R. M., Schramm, S. M. (2013). Optimization and stability of the contrast transfer function in aberration-corrected electron microscopy. Ultramicroscopy, (125): 72. https://doi.org/10.1016/j.ultramic.2012.09.007

Urban, K. W. (2008). Studying atomic structures by aberration-corrected transmission electron microscopy. Science, (321):506.https://doi.org/10.1126/science.1152800

Van Tendeloo, G., Bals, S., Van Aert, S., Verbeeck, J., Van Dyck, D. (2012). Advanced electron microscopy for advanced materials. Adv. Mater., (24): 5655. https:// doi.org/10.1002/adma.201202107

Vulovic, M., Franken, E., Ravelli, R. B. G., Van Vliet, L. J., Rieger, B. (2012). Precise and unbiased estimation of astigmatism and defocus in transmission electron microscopy. Ultramicroscopy, (116): 115. https://doi.org/10.1016/j.ultramic.2012.03.004

Wen-Kuo, H., Fu-Rong, Ch., Ji-Jung, K., Kirkland, A. I. (2004). Resolution extension and exit wave reconstruction in complex HREM. Ultramicroscopy, (98): 99. https://doi.org/10.1016/j.ultramic.2003.08.004

Yang, C., Jiang, W., Chen, D. H., Adiga, U., Ng, E. G., Chiu, W. (2009). Estimating contrast transfer function and associated parameters by constrained non-linear optimization. Journal of Microscopy, (233): 391. https://doi.org/10.1111/ j.1365-2818.2009.03137.x

Young-Min, K., Jong-Man, J., Jin-Gyu, K., Youn-Joong, K. (2006). Image processing of atomic resolution transmission electron microscope images. J. Korean Phys. Soc., (48): 250. https://www.springer.com/journal/40042

Zhu, J., Penczek, P. A., Schröder, R., Frank, J. (1997). Three-dimensional reconstruction with contrast transfer function correction from energy-filtered cryoelectron micrographs: Procedure and application to the 70S Escherichia coli ribosome. J. Struct. Biol., (118): 197. https://doi.org/10.1006/jsbi.1997.3845 
